\title{
Pharmacotherapy for Weight Management in the VHA
}

\author{
Todd P. Semla, MS, PharmD ${ }^{1,2}$, Chris Ruser, MD ${ }^{3,4}$, Chester B. Good, MD, MPH 1,5,6, \\ Susan Z. Yanovski, $M D^{7}$, Donna Ames, $M D^{8,9}$, Laurel A. Copeland, $P h D^{10}$, Charles Billington, $M D^{17}$, \\ U. Inge Ferguson, $\mathrm{DO}^{12}$, Louis J. Aronne, $\mathrm{MD}^{13}$, Thomas A. Wadden, $\mathrm{PhD} \mathrm{D}^{14}$, \\ W. Timothy Garvey, $\mathrm{MD}^{15,16}$, Caroline M. Apovian, $\mathrm{MD}^{17}$, and David Atkins, MD, $\mathrm{MPH}^{18}$
}

\begin{abstract}
'Pharmacy Benefits Management Services, Department of Veterans Affairs, Hines, IL, USA; ${ }^{2}$ Department of Medicine, and Psychiatry and Behavioral Sciences, Northwestern University Feinberg School of Medicine, Chicago, IL, USA; ${ }^{2}$ VA Connecticut Healthcare System, New Haven, CT, USA; ${ }^{4}$ Yale University School of Medicine, New Haven, CT, USA; ${ }^{5}$ Center for Health Equity Research and Promotion, VA Pittsburgh Healthcare System, Pittsburgh, PA, USA; ${ }^{6}$ Division of General Medicine, University of Pittsburgh School of Medicine, Pittsburgh, PA, USA; ${ }^{7}$ National Institute of Diabetes and Digestive and Kidney Diseases, National Institutes of Health, Bethesda, MD, USA; ${ }^{8}$ David Geffen School of Medicine, University of California, Los Angeles, CA, USA; ${ }^{2}$ West Los Angeles VA Medical Center, Los Angeles, CA, USA; ${ }^{10}$ VA Central Western Massachusetts, Leeds, MA, USA; " Minneapolis VA Medical Center, Minneapolis, MN, USA; ${ }^{2}$ VA Southern Nevada Health Care System, Las Vegas, NV, USA; ${ }^{13}$ Comprehensive Weight Control Program, Weill-Cornell Medicine, New York, NY, USA; ${ }^{14}$ Center for Weight and Eating Disorders, Perelman School of Medicine, University of Pennsylvania, Philadelphia, PA, USA; ${ }^{15}$ Department of Nutrition Sciences, University of Alabama at Birmingham, Birmingham, AL, USA; ${ }^{16}$ Geriatric Research, Education and Clinical Center, Birmingham VA Medical Center, Birmingham, AL, USA; ${ }^{17}$ Boston University School of Medicine, Nutrition and Weight Management and Nutrition and Support Service at Boston Medical Center, Boston, MA, USA; ${ }^{18} \mathrm{Health}$ Service Research and Development, US Department of Veterans Affairs, Washington, DC, USA.
\end{abstract}

Weight management medications (WMM) are underutilized as an adjunct to behavioral and lifestyle interventions. In fiscal years 2014-2015, a total of approximately 2500 veterans-a mere $2 \%$ of veterans receiving care from the Veterans Health Administration (VHA)—eligible for a WMM received a prescription for one. A State of the Art Conference on Weight Management workgroup, focused on pharmacotherapy, developed evidence-based recommendations and strategies to foster the appropriate use of WMM in the VHA. The workgroup identified patient, prescriber, and health system barriers to and facilitators for prescribing WMM. Barriers included patient and provider concerns about medication safety and efficacy, limited involvement of primary care, restrictive medication criteria for use (CFU), and skepticism among providers regarding the safety and efficacy of WMM and the perception of obesity as a disease. Potential facilitators for removing barriers included patient and provider education about WMM and the health benefits of weight loss, increased engagement of primary care providers in weight management, relaxation of the CFU, and creation of a system to help patients navigate through weight management treatment options. Several research questions were framed with regard to WMM in general, and specifically to the care of obese veterans. While some of the workgroup's conclusions reflect issues specific to the VHA, many are likely to be applicable to other health organizations.

KEY WORDS: obesity; pharmaceutical care; veterans.

J Gen Intern Med 32(Suppl 1):S70-S3

DOI: $10.1007 / \mathrm{s} 11606-016-3949-\mathrm{y}$

(c) Society of General Internal Medicine 2016

\section{BACKGROUND}

The obesity epidemic among U.S. veterans likely exceeds that in the general population. Forty percent of veterans

Published online March 7, 2017 receiving care from the Veterans Health Administration (VHA) are obese (a BMI of $30 \mathrm{~kg} / \mathrm{m}^{2}$ or greater), and another $38.5 \%$ are overweight (a BMI $\geq 25 \mathrm{~kg} / \mathrm{m}^{2}$ and $<30 \mathrm{~kg} / \mathrm{m}^{2}$ ) - a total of nearly four million veterans. ${ }^{1}$ The potential health implications of this epidemic for veterans and the associated costs for the VHA are staggering. And, as with the general population, the traditional medical model has failed to offer successful long-term treatment. One reason for this failure is a perceived lack of safe and effective pharmacotherapy, likely attributable to the complex etiology of excess weight, overlapping appetite regulation systems in the human body, and the infamous history of weight management medications (WMM).

This pattern is borne out in recent VHA statistics. In fiscal years (FY) 2014 and 2015, 2508 and 2528 veterans receiving care from the VHA were prescribed a Food and Drug Administration (FDA)-approved WMM (orlistat, phentermine, phentermine/topiramate, or lorcaserin), respectively. That equates to less than $2 \%$ of the 135,000 veterans seen in the VHA's MOVE! weight management program in FY2015, and less than $0.1 \%$ of veterans eligible for MOVE!. ${ }^{2,3}$ Not only has the use of WMM been low in the recent past; it has actually decreased since 2010 , when nearly 4000 veterans were prescribed a WMM (mostly orlistat). ${ }^{4}$ Given the prevalence of overweight and obesity among veterans, it would thus appear that WMM are underutilized within the VHA. This pattern of underuse is surprising, considering the widespread availability of a supportive behavioral program, MOVE!, and the recent FDA approval of four WMM.

Underuse of WMM, however, is not limited to the VHA. A recent national study found that only $1 \%$ of obese U.S. individuals filled a prescription for a weight management drug 
over a 4-year period. ${ }^{5}$ And between 1991 and 2011, with the exception of phentermine, the prescribing of WMM declined. ${ }^{6,7}$ The absence of physician training in pharmacotherapy for obesity, coupled with impediments in our health care system (lack of insurance coverage for WMM, limited patient-provider time for discussing interventions), limit providers' ability to achieve optimal outcomes in patients with obesity. Conversely, it is estimated that $30 \%$ of obese U.S. adults have used dietary supplements for weight loss, resulting in more than 23,000 emergency department visits annually. ${ }^{8,9}$ The underuse of effective drugs and overuse of unproven supplements for weight loss underscores the need for a reappraisal of pharmacotherapeutic strategies for combating obesity.

Clinical practice guidelines for the management of overweight and obese adults, including those put forth by the VHA and Department of Defense, are in agreement that the place of pharmacotherapy in weight management should follow FDA label indications. Pharmacotherapy is recommended as an adjunct to behavior and lifestyle interventions. ${ }^{1,10-12}$

None of the WMM are on the VA National Formulary (VANF). All drugs are available through the non-formulary drug request process (i.e., prior authorization), provided that the veteran meets the requested drug's criteria for use (CFU). Common to all CFU are that the veteran must 1) meet the FDA label indications with respect to $\mathrm{BMI} \pm$ the presence of comorbidities, 2) be enrolled and participating in MOVE!, and 3) achieve a specific weight loss criterion (often found in the drug's labeling) in order to continue the medication. The absence of a WMM on the VANF is not unique to the VHA, and likely reflects their cost, perceived benefit, and safety concerns. In the VHA, the employment of CFU is an effort to limit access to WMM to those patients most likely to benefit, while at the same time containing costs.

\section{HSR\&D STATE OF THE ART CONFERENCE ON WEIGHT MANAGEMENT}

A VHA Weight Management State of the Art Conference (SOTA) was designed to bring together VHA and non-VHA experts in weight management, with the goal of synthesizing what we know and what we need to know about weight management within the VHA and the larger health care community. Specifically, the SOTA addressed patient-, provider-, and system-level barriers and facilitators to bariatric surgery, comprehensive lifestyle interventions, and approved pharmacotherapies. The SOTA sought consensus where evidence was found to be sufficient and identified gaps where evidence was conflicting or lacking. It sought to define and develop a research agenda to address conflicts/ gaps. Also, based on the evidence, the SOTA participants were tasked with drafting recommendations/strategies to assist the VHA in implementing and improving weight management programs and outcomes. Pharmacotherapy was one of three workgroups convened as part of the SOTA; the other two addressed behavioral and surgical interventions. The workgroup comprised ten physicians (seven experts in obesity, two internists, and one psychiatrist), two psychologists, and a pharmacist from within and outside the VHA. The workgroup was charged with considering four key questions regarding pharmacotherapy for weight management:

1. What are the patient and health system barriers and facilitators to prescribing WMM for obese VHA patients?

2. In which patients has the addition of WMM proven effective in enhancing weight loss compared to standard care?

3. What is the average effect of WMM on weight? What factors affect the selection of a WMM for a patient?

4. What are the key research questions the VHA should address regarding WMM for weight management?

The workgroup was given a reading list of literature relevant to the topic. We conducted six conference calls to address each key question in preparation for the 2-day SOTA, which convened all three workgroups to share their findings and formulate recommendations.

For key question \#1, the workgroup identified concerns about medication safety and efficacy, confusion about the role of medication relative to other therapies, and questions about the individual contraindications for each medication as patient barriers. Factors that were considered potential facilitators of patient acceptance were education regarding available medications and a shared decision-making approach to selecting treatment options. Health system barriers included overly restrictive $\mathrm{CFU}$ and inconsistent interpretation across the VHA, and a lack of universal acceptance of obesity as a chronic disease. Strategies for enhancing appropriate prescribing by VHA providers included the following: revising the $\mathrm{CFU}$ to be more flexible as to when a drug could be initiated-specifically, sooner than the required 90-day enrollment in MOVE!; educating health care professionals and administrators regarding the characterization of obesity as a chronic condition requiring longterm management; developing and instituting better identification and tracking of patients who do not respond to behavioral and lifestyle interventions; and compiling data on the costs of pharmacotherapy relative to the benefits of additional weight loss. A critical, intrinsic benefit to the VA system with regard to use of adjunctive WMM is the widespread availability of a standardized behavioral weight loss program, MOVE!. This program provides a large-scale multidisciplinary context to support prescribing by primary care practitioners, as opposed to non-VA systems, in which availability of adjunctive behavioral programs may be inconsistent or unavailable.

For key question \#2, the workgroup was unanimous in its view that WMM should be prescribed only for patients who 
meet the FDA labeling indications, including minimum BMI requirements, and should not be used in isolation, but rather as an adjunct to behavioral and lifestyle modifications. The workgroup agreed that it was not possible to predict which patients would respond to WMM or to a specific medication. However, those who did not respond would be detectable early in the course of treatment, e.g., by 12 weeks, and such patients should discontinue the medication, be re-evaluated, and be considered for another medication or alternative or enhanced treatment modalities. ${ }^{1,10-12}$

Key question \#3 was addressed, in part, using the findings from clinical trials, systematic reviews and metaanalyses. ${ }^{13-27}$ At 12 months after initiation of treatment, adjunct pharmacotherapy resulted in moderate additional weight loss compared to behavioral and lifestyle interventions alone, with a significantly greater proportion of patients losing 5 or $10 \%$ of total body weight. These findings were recently confirmed in another systematic review and metaanalysis. ${ }^{28}$ Ranking the drug products by mean placebosubtracted weight loss and odds of achieving $\geq 5 \%$ weight loss compared to placebo suggests that a phenterminetopiramate combination results in the greatest weight loss $(-8.8 \mathrm{~kg}$; OR 9.1, 95\% CI 7.68-10.78) and orlistat the least $(-2.63 \mathrm{~kg}$; OR $2.69,95 \%$ CI $2.36-3.07) .^{28}$ Comorbidities and drug-drug interactions are factors that influence drug selection, e.g., avoiding phentermine in patients with hypertension and avoiding lorcaserin in patients taking drugs affecting serotonin or dopamine. The Risk Evaluation and Mitigation Strategy (REMS) program for phentermine/ topiramate initially limited veterans' access to the product, as it utilized specialty pharmacy dispensing. Access was increased when VHA pharmacies became eligible to dispense the combination product.

The VHA CFU for weight management medications requires that a veteran be enrolled and an active participant in MOVE! for at least 3 months and that the patient's weight loss with diet, exercise, and behavioral intervention has stopped or plateaued. The workgroup concluded that these requirements were not evidence-based. In the phase 3 clinical trials, medication was started simultaneously with diet and lifestyle intervention. Hence, if following the VHA $\mathrm{CFU}$, the weight loss realized by veterans may not be of the same magnitude at 12 weeks as that recommended by a drug's product label. There are other potential reasons that degree of weight loss achieved by veterans may be different from that seen in clinical trials, including demographic differences between populations (e.g., VHA population less likely to be female).

Key question \#4 identified three areas of research need. The first is identifying patient factors that predict response to WMM, including age, gender, level of obesity, comorbidities, and previous weight loss history and interventions. The second is collecting data on outcomes after more than 2 years of weight management intervention, including quality of life, effects on comorbidities (e.g., cardiovascular and diabetes outcomes), and medication safety, in order to analyze the benefits, harms, and cost effectiveness of weight management pharmacotherapy. The third need is studying the impact of the unique weight management problems faced by veterans, including mental health conditions such as PTSD and high levels of concomitant psychotropic medications use. ${ }^{29}$ In addition, studies in the veteran population are needed to identify the best starting point for introducing pharmacotherapy, the most effective adjunctive behavioral treatments, and the role of intermittent vs. continuous therapy as well as duration of treatment.

\section{RECOMMENDATIONS TO INCREASE APPROPRIATE USE OF WEIGHT MANAGEMENT MEDICATIONS IN VHA}

The workgroup provided several recommendations to facilitate the appropriate prescribing of WMM. Some of these can be implemented in a short time frame through changes in policy and guidance, while others require education and outreach to both patients and health care providers. The VHA's Pharmacy Benefits Management (PBM) has revised the CFU for all WMM by eliminating the requirement that the veteran be enrolled in MOVE! for at least 3 months and that their weight loss has plateaued prior to starting a drug. Instead, veterans must be participating in a clinically supported weight management program that targets all three aspects of weight management (i.e., diet, physical activity, behavioral changes) through MOVE! or a similar, nonVHA program. The intent is to allow prescribers to start a medication when the patient is ready, to recognize that MOVE! programs cannot accommodate all veterans interested in losing weight, and that not all MOVE! programs have access to physicians who can prescribe WMM. The change would also allow more primary care and specialty providers (endocrinologists, psychiatrists) to prescribe WMM to veterans. Clinical pharmacists in the VHA have expanded their scope of practice, and many are involved in MOVE! or other specialty clinics, with a large proportion practicing in primary care as members of the Patient Aligned Care Team (PACT). A potential role for PACT pharmacists would be to serve as a liaison between PACT physicians (prescribers) and MOVE! or patients utilizing non-VA weight management programs/resources.

It is not known what the actual demand for weight management pharmacotherapy among veterans would be if there were improved access to treatment and fewer barriers to prescription, nor is it known how changes in the CFU will impact prescribing. In both the VHA and the private sector, obesity has deleterious effects that permeate patients' lives and tax health care resources. There is no magic pill to melt away the pounds; however, adjunctive pharmacotherapy provided through a supportive and collaborative effort between patient, pharmacist, and medical and behavioral 
health care providers can enhance the likelihood of clinically meaningful weight loss and improved health.

Acknowledgments: The Weight Management SOTA was funded by the VA Health Services Research and Development Service. All commentary and conclusions are those of the authors, and do not necessarily represent the opinions of the Health Services Research and Development Service or the Department of Veterans Affairs.

Corresponding Author: Todd P. Semla, MS, PharmD; Pharmacy Benefits Management ServicesDepartment of Veterans Affairs, 1st Ave. -1 Block N. of Cermak Rd, Building 37 (1OP4P), Hines, IL 60141, USA (e-mail: Todd.semla@va.gov).

\section{Compliance with Ethical Standards:}

Conflict of Interest Summary: Todd P. Semla, MS, PharmD spouse is an employee of Abbvie and formerly of Abbott Labs, and owns stock in both companies. Chris Ruser, MD - none. Chester B. Good, MD, MPH - none. Susan Z. Yanovski, MD - spouse receives research project support from Zafgen and Rhythm Pharmaceuticals. Donna Ames, MD - none. Laurel A Copeland, PhD Mallinckrodt (grant support). Charles Billington, MD - Novo Nordisk. U. Inge Ferguson, DO - none. Louis Aronne, MD - Stock ownership: BMIQ, Zafgen Inc., ERx, MYOS Corporation; Consultancies: Johnson \& Johnson, Ethicon Endo-Surgery Inc., VIVUS, Inc., Novo Nordisk A/S, GI Dynamics; Grants received: Eisai, Inc., Astra Zeneca. Thomas Wadden, PhD - serves on advisory boards for Weight Watchers, Nutrisystem, and Novo Nordisk. Tim Garvey, MD - Consultancies: Novo Nordisk, Janssen, Alexion, VIVUS, Eisai, Takeda, AstraZeneca, Merck; Research sponsored by institution (UAB) with Dr. Garvey as site PI: Merck, AstraZeneca, Weight Watchers, Eisai, Pfizer, Novo Nordisk, Lexicon, Elcelyx; Stock ownership (all publically traded): Eli Lilly, Pfizer, Novartis, Merck, Isis, Bristol-Myers-Squibb. Caroline Apovian, MD - Consulting: Merck, Johnson \& Johnson, Arena Nutrisystem, Zafgen, Sanofi-Aventis, Orexigen, EnteroMedics, Scientific Intake, Gelesis, Takeda Pharmaceuticals U.S.A., Novo Nordisk; Stock ownership: Science Smart LLC; Research funding: Lilly, Aspire Bariatrics, GI Dynamics, Pfizer, Sanofi-Aventis, Orexigen, MetaProteomics, the Dr. Robert C. and Veronica Atkins Foundation, and MYOS Corporation. David Atkins, $M D, M P H$ - none.

\section{REFERENCES}

1. Department of Veterans Affairs and Department of Defense. VA/DoD Clinical Practice Guideline for Screening and Management of Overweight and Obesity, version 2.0, 2014. http://www.healthquality.va.gov/guidelines/ CD/obesity/CPGManagementOfOverweightAndObesityFINAL041315.pdf Accessed July 17, 2016.

2. Kahwati L, Trang XL, Jones KR, Kinsinger LS. RE-AIM evaluation of the Veterans Health Administration's MOVE! Weight Management Program. Transl Behav Med. 2011;1:551-60.

3. U.S. Department of Veterans Affairs MOVE! Weight Management Program. Available at: http://www.move.va.gov/. Accessed July 22, 2016.

4. Data from the Pharmacy Benefits Management Services (PBM) prescription database (Hines, IL). July 21, 2016.

5. Bessesen DH, McCormick E, Saxon DR, et al. Patterns of prescribing of weight loss medications in a large cohort of adults. LB-OR02-05 Presented at the ENDO2016, The Endocrine Society Annual Meeting. Boston, MA, April 3, 2016. Available at: https://endo.confex.com/endo/ 2016endo/webprogram/Paper28282.html Accessed July 21, 2016.

6. Hampp C, Kang EM, Borders-Hemphill v. Use of prescription antiobesity drugs in the United States. Pharmacotherapy. 2013;33(12):1299-307.

7. Block JP, Choudhry NK, Carpenter DP, et al. Time series analyses of the effect of FDA communications on use of prescription weight loss medications. Obesity. 2014;22(3):943-9.

8. Pillitteri JL, Shiffma S, Rohay JM, Harkins AM, Burton SL, Wadden TA. Use of dietary supplements for weight loss in the United States: results of a national survey. Obesity. 2008;16:790-6.
9. Geller AI, Shehab N, Weidle NJ, et al. Emergency department visits for adverse events related to dietary supplements. N Engl $\mathrm{J}$ Med. 2015;373:1531-40.

10. Yanovski SZ, Yanovski JA. Long-term drug treatment for obesity: a systematic and clinical review. JAMA. 2014;31(1):74-86.

11. Ollendorf DA, Cameron CG, Pearson SD. Effectiveness and value of treatment options for obesity-a report for the California technology assessment forum. JAMA Intern Med. 2016;176(2):247-8.

12. Garvey WT, Mechanick JI, Brett EM, Garber AJ, Hurley DL, Jastreboff AM, Nadolsky K, Pessah-Pollack R, Plodkowski R; Reviewers of the AACE/ACE Obesity Clinical Practice Guidelines. American Association of Clinical Endocrinologists and American College of Endocrinology Clinical Practice Guidelines for Comprehensive Medical Care of Patients with Obesity. Endocrine Pract. In Press, 2016. Epub May 24, 2016. doi:10.4158/EP161365.GL.

13. Apovian CM, Aronne LJ, Bessesen DH, et al. Pharmacologic management of obesity: an Endocrine Society clinical practice guideline. J Clin Endocrinol Metab. 2015;100:342-62.

14. Jensen MD, Ryan DH, Apovian CM, et al. 2013 AHA/ACC/TOS guideline for the management of overweight and obesity in adults: a report of the American College of Cardiology/American Heart Association Task Force on Practice Guidelines and The Obesity Society. J Am Coll Cardiol. 2014:63:2985-3023.

15. Smith SR, Weissman NJ, Anderson CM, et al. Multicenter, placebocontrolled trial of lorcaserin for weight management. N Engl J Med. 2010;363:245-56.

16. O'Neil PM, Smith SR, Weissman NJ, et al. Randomized placebocontrolled clinical trial of lorcaserin for weight loss in type 2 diabetes mellitus: the BLOOM-DM study. Obesity. 2012;20:1426-36.

17. Fidler MC, Sanchez M, Raether B, et al. A one-year randomized trial of lorcaserin for weight loss in obese and overweight adults: the BLOSSOM trial. J Clin Endocrinol Metab. 2011;96:3067-77.

18. Allison DB, Gadde KM, Garvey WT, et al. Controlled-release phentermine/topiramate in severely obese adults: a randomized control trial (EQUIP). Obesity. 2011;20:330-42.

19. Gadde KM, Allison DB, Ryan DH, et al. Effects of low-dose, controlledrelease, phentermine plus topiramate combination on weight and associated comorbidities in overweight and obese adults (CONGUER): a randomized, placebo-controlled, phase 3 trial. Lancet. 2011;377:1341-52.

20. Pi-Sunyer X, Astrup A, Fujioka K, Greenway F, Halpern A, et al. Randomized, controlled trial of $3.0 \mathrm{mg}$ of Liraglutide in weight management. N Engl J Med. 2015;373:11-22.

21. Astrup A, Rossner S, Van Gaal L, et al. Effects of liraglutide in the treatment of obesity: a randomized, double-blind, placebo-controlled study. Lancet. 2009;374:1606-16.

22. Wadden TA, Hollander $\mathbf{P}$, Klein $\mathbf{S}$, et al. Weight maintenance and additional weight loss with liraglutide after low-calorie-diet-induced weight loss: the SCALE Maintenance randomized study. Int $\mathrm{J}$ Obes. 2013;37:1443-51.

23. Yanovski SZ, Yanovski JA. Naltrexone extended-release plus bupropion extended-release for treatment of obesity. JAMA. 2015;313(12):1213-4.

24. Greenway FL, Fujioka K, Plodkowski RA, et al. COR-I Study Group. Effect of naltrexone plus bupropion on weight loss in overweight and obese adults (COR-I). Lancet. 2010;376(9741):595-605.

25. Apovian CM, Aronne L, Rubino D, et al. COR-II Study Group. A randomized, phase 3 trial of naltrexone SR/bupropion SR on weight and obesity-related risk factors (COR-II). Obesity (Silver Spring). 2013;21(5):935-43.

26. Wadden TA, Foreyt JP, Foster GD, et al. Weight loss with naltrexone SR/bupropion SR combination therapy as an adjunct to behavior modification: the COR-BMOD trial. Obesity (Silver Spring). 2011;19(1):110-20.

27. Hollander P, Gupta AK, Plodkowski R, et al. COR-Diabetes Study Group. Effects of naltrexone sustained-release/bupropion sustainedrelease combination therapy on body weight and glycemic parameters in overweight and obese patients with type 2 diabetes. Diabetes Care. 2013;36(12):4022-9.

28. Khera R, Murad MH, Chandar AK, et al. Association of pharmacological treatments for obesity with weight loss and adverse events: a systematic review and meta-analysis. JAMA. 2016;315:2424-34.

29. Ames D, Carr-Lopez SM, Gutierrez MA, et al. Detecting and managing adverse effects of antipsychotic medications. Psychiatr Clin North Am. 2016:39:275-311. 